\title{
Pflegelücke in Deutschland \\ Es geht nicht um sauber, satt und freundlich!
}

Das Image der Pflegeberufe ist in der Gesellschaft antiquiert und unattraktiv. Aber für Irene Maier, Vorstandsvorsitzende des Verbandes der Pflegedirektorinnen und Pflegedirektoren der Universitätskliniken und Medizinischen Hochschulen Deutschlands e.V. (VPU), ist der Pflegeberuf besser als sein Ruf. Bislang habe man nur nicht vermittelt können, wie anspruchsvoll dieser Beruf sei und welche intellektuellen Anforderungen er mit sich bringt. Hier gehe es um mehr als um sauber, satt und freundlich, und Irene Maier weiß wovon sie spricht. Seit über 20 Jahren ist sie im Bereich Pflege Irene Maier tätig: nach Stationen als Pflegedienstleitung und Pflegedirektorin am Universitätsklinikum Lübeck, ist sie seit 1996 als Direktorin für die pflegerische Versorgung der Patienten am Universitätsklinikum Essen verantwortlich. Im vergangenen November war sie Kongresspräsidentin des 34. Deutschen Krankenhaustages in Düsseldorf.

? In Deutschland gehen den Krankenhäusern die Pflegekräfte aus. Kennt man genaue Zahlen?

Irene Maier: Verlässliche Zahlen kann ich nicht nennen. Ich gehe davon aus, dass wir zwischen 3000 und 5000 offene Stellen haben, die nicht besetzt werden können, und ich kenne kein Krankenhaus, das nicht Mühe hat, diese offenen Stellen zu besetzen.

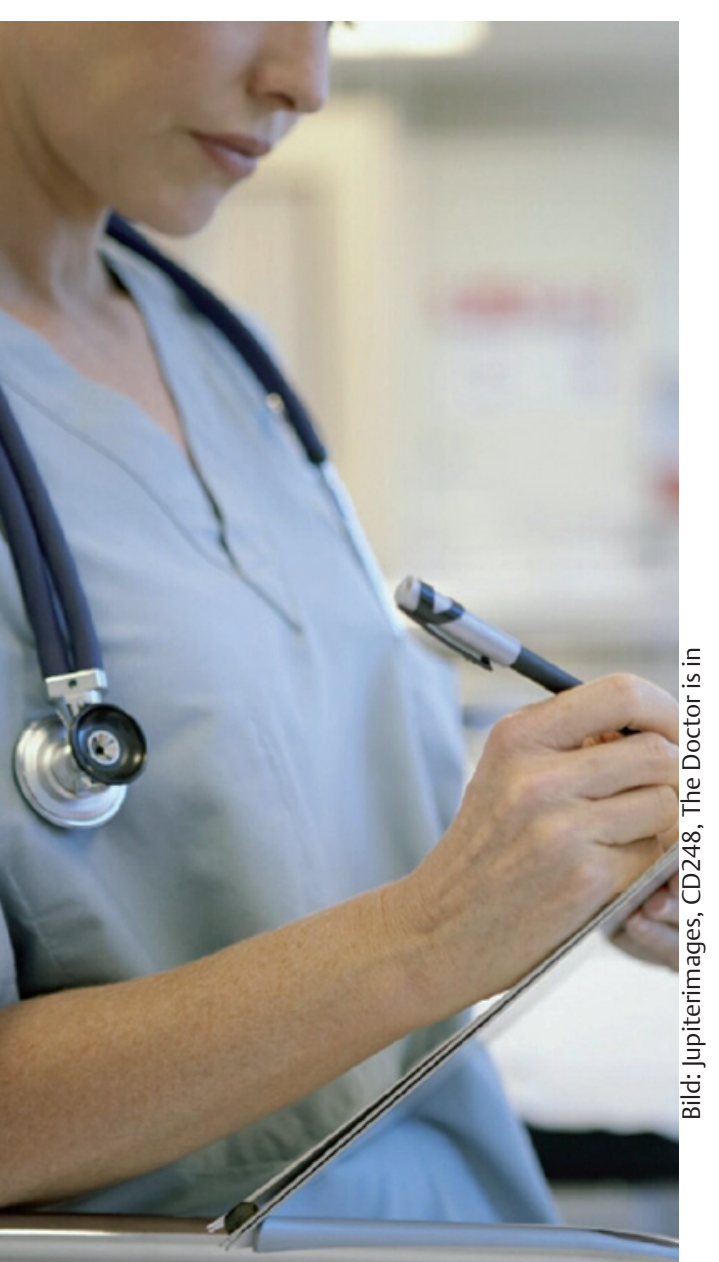

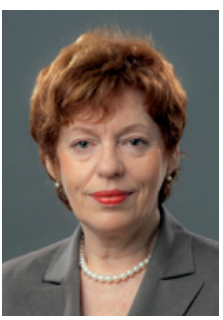

steigenden Bedarf an Pflegekräften zu errechnen?

Irene Maier: Das kann man nicht eins-zueins umsetzen. Wir werden andere Arbeitsorganisationen brauchen. Die Fachkraft steht sehr wohl am Anfang, aber durchaus auch Angehörige oder Hilfskräfte.

? Wo soll der Nachwuchs herkommen, um diese Pflegelücke zu schließen? Irene Maier: In erster Linie aus unserer Jungbevölkerung, die wir für den Pflegeberuf interessieren müssen. Wir haben hier in Essen ein Projekt mit Gymnasialschülern durchgeführt „Jugend denkt Zukunft" und das hat bestätigt: die Schüler wissen kaum etwas über diesen Beruf. Sie haben keine Vorstellung davon, welche Spezialisierungsmöglichkeiten er bietet und welche intellektuellen Anforderungen er mit sich bringt. Da liegt der Fehler, das muss transportiert werden. Aber mit dem Nachwuchs alleine werden wir das Problem nicht lösen können. Wir brauchen auch andere Programme, damit gut ausgebildete Mitarbeiter mit Facherfahrung, die pausieren, zurückkommen. Dafür braucht es flexiblere Arbeitszeiten, mehr Integration von Familie und Beruf.

Das ist meine Prognose.

? Und wo fehlen sie aktuell? Ist heute auch schon die gesamte, ja sehr unterschiedliche Krankenhauslandschaft betroffen?

Irene Maier: Sie fehlen derzeit vor allem in den Spezialabteilungen wie der Onkologie, den OPs, in der Anästhesie oder auf den Intensivstationen. Da wird der Pflegefachkräftemangel sichtbar und das in Ballungsgebieten noch stärker als in ländlichen Regionen. Und das zeigt uns zum einen, dass das Fachpersonal, wenn es z.B. in die Elternzeit geht, nicht mehr zurückkommt. Oder dass wir das Fachpersonal an die Industrie verlieren. Gut ausgebildete Leute erhalten in der Industrie attraktive Angebote, die sie verständlicherweise auch annehmen.

? Das Statistische Bundesamt hat ausgerechnet, dass die Zahl der Pflegebedürftigen im Jahr 2020 auf 2,9 Millionen steigen wird. Ist das ein verlässlicher Schlüssel, um den
Heißt das, die Kliniken sind zu unflexibel, um den erfahrenen Fachkräften den Wiedereinstieg schmackhaft zu machen?

Irene Maier: Ja, ich glaube sie sind mit ihren Programmen noch nicht flexibel genug, um auf diese Anforderung entsprechend zu reagieren.

Es gibt in der Klinik neben der examinierten Pflegekraft die gelernte Hilfskraft und es gibt Kliniken, die in der Weiterbildung und Förderung dieser Hilfskraft durchaus die Lösung des Versorgungsdefizits sehen. Halten Sie das für eine gute Lösung, die nicht auf Kosten der Pflegequalität geht? Irene Maier: Es ist sicher richtig, wenn man die Krankenhausorganisation mit einem anderen Personal-Mix aufbaut: dazu gehören die dreijährig Ausgebildeten, Pflegeassistenten, aber auch akademisch ausgebildete Mitarbeiter. Wenn es angelernte Kräfte gibt, die sich für die Pflege- 
assistentenausbildung eignen, dann ist das ein richtiger Weg, und der muss nicht zu Qualitätseinbußen führen.

Das Image der Pflegeberufe ist eher unattraktiv - Bezahlung mäßig und Arbeitsbedingungen schlecht. Ist der Pflegeberuf besser als sein Ruf? Irene Maier: Auf jeden Fall! Wenn man mit Pflegekräften spricht, die schon lange im Beruf sind, dann spürt man die Zufriedenheit. Was fehlt, ist die gesellschaftliche Anerkennung. Das Pflegeberufsbild ist in der Gesellschaft zu antiquiert. Bislang konnte nicht wirklich vermittelt werden, welch anspruchsvoller Beruf mit intellektuellen Anforderungen das ist. Vielmehr hat die Gesellschaft immer noch das Gefühl, es geht um sauber, satt und freundlich.

Aber ist es auch ein Beruf in dem man weiterkommt? Oder ist mit der dreijährigen Generalausbildung das Ende der Fahnenstange erreicht? Irene Maier: Nach der Grundausbildung geht es mit der Spezialisierung für unterschiedliche Fachrichtungen eigentlich erst richtig los. Da sind die Fachweiterbildungen beispielsweise für den $\mathrm{OP}$, die Anästhesie, die Dialyse, für die Onkologie usw.

\section{? ... und lohnen sich solche Speziali-} sierungen?

Irene Maier: Da fängt das Problem an dieser Aufstieg ist kein finanzieller! Das ist ein Stufenanstieg von nicht mehr als 250 €uro brutto. Das macht eine zweijährige Fachweiterbildung mit immerhin 720 Stunden Theorie und einer zweijährigen Rotation in der Praxis wenig attraktiv. Von der finanziellen Seite lohnt sich der Einsatz nicht.

Ist unter diesem Aspekt die jetzige, dreijährige Ausbildung noch zeitgemäß?

Irene Maier: Nein, sie ist veraltet! Aber die Generalisierung braucht man, weil das der Alltag der Versorgung ist. Wir haben heute Patienten auf den Stationen, die aus den Altenheimen kommen und dement sind. D.h. die Altenpflege ist absolut auch ein Tätigkeitsfeld im Krankenhaus und umgekehrt.

Wenn die dreijährige Ausbildung eine Sackgasse ist, wie kann man im Pflegeberuf denn dann Karriere machen? Gibt es andere Aufstiegschancen?
Irene Maier: Ich kenne kaum einen Beruf, der so viele Aufstiegsmöglichkeiten bietet. Man kann sich beispielsweise akademisch weiterbilden - ob im pflegewissenschaftlichen und pflegepädagogischen Bereich, oder auf der Managementebene bis hin zur Promotion und Habilitation.

Was könnte die anstehende Reform durch den Staat der Ausbildung bringen? Würde die Eingliederung in das staatliche Bildungssystem die Pflegeausbildung attraktiver machen? Irene Maier: Das wäre auf jeden Fall so. Wenn diese Ausbildung mit anderen Berufsausbildungen egalisiert würde und wir eine andere Finanzierungsgrundlage haben, macht das den Beruf attraktiver. Und die Schüler würden nicht, wie heute üblich, als volle Arbeitskraft gelten.

\section{... und was kann der Gesetzgeber} noch tun, um den Pflegeberuf attraktiver $\mathrm{zu}$ machen?

Irene Maier: Der Gesetzgeber ist dafür verantwortlich, wie eine Ausbildungs- und Prüfungsverordnung aussieht und hat damit auch in der Hand, wie attraktiv ein Beruf ist. Er muss die aus der Praxis bekannten Inhalte, wie die unterschiedlichen Versorgungsfelder mit in das Gesetz aufnehmen und er muss die Vorbehaltsaufgaben regeln. Ganz wichtig - das war auch die Forderung der jungen Leute in unseren Projektwochen in Essen - ist ein Auslandssemester als Standard. Junge Leute wollen Auslandspraktika machen können; sie wollen eine internationale Ausbildung, die für sie auch die Attraktivität des Pflegeberufes steigern würde. Für wichtig halte ich auch, dass man die Gesundheitsfachberufe bei bestimmten Inhalten zusammen ausbildet; dass die Medizinstudenten mit den Auszubildenden in den Gesundheitsfachberufen gemeinsame Fachmodule haben.

Sehen Sie denn bei der Politik den Wille zu solchen Reformen? Irene Maier: Nein, kann ich nicht erkennen!

\section{Das Generalthema des letzten} Deutschen Krankenhaustages, dessen Präsidentin sie waren, hieß „Versorgung verbessern - Barrieren abbauen!“. Sie werfen der Gesundheitspolitik vor, dass dieses Versorgungsstrukturgesetz massive inhaltliche Versäumnisse hat. Was fehlt diesem Gesetz, um die Situation zu verbessern?
Irene Maier: Für mich hatte das Gesetz die eindeutige Anforderung, dass die Aufgabenfelder neu geordnet werden müssen. Was die Aufgabenveränderung der Gesundheitsfachberufe betrifft, springt das Gesetz nicht weit genug nach vorne. Das gilt nicht nur für die Pflegeberufe, sondern auch für die Physiotherapie, oder die Logopäden. Da ist Expertise vorhanden, die im Sinne von Eigenverantwortlichkeit und Anordnungsfähigkeit ins Gesetz eingebracht hätte werden können. Das nimmt das Gesetz nicht auf und das ist ein Manko!

Würde die Neuverteilung bzw. Übertragung von Aufgaben die Situation entschärfen?

Irene Maier: Ich bin davon überzeugt, dass man auf diesem Wege eine Entschärfung der Problematik erreichen kann. Leider ist das häufigste Missverständnis die Befürchtung, dass die Krankenschwester ein Pseudomediziner werden will. Das ist aber nicht gemeint, sondern dass fachweitergebildete Pflegekräfte bestimmte Schritte an chronisch kranken Patienten vorbereiten, z.B. beim Diabetes, im Wundmanagement oder der Herzinsuffizienz, ohne dass der Arzt eingeschaltet werden muss und hier entlasten können.

Dann müsste aber zuerst definiert werden, wo die ärztliche Tätigkeit beginnt und die der Pflegekräfte endet. Wer soll das machen - der Gesetzgeber? Irene Maier: Das kann ich aus dem Stand nicht definieren, denn das ist ein sensibler Prozess, den man mit der Ärzteschaft gemeinsam gehen muss. Es muss geklärt werden, bis zu welcher Schnittstelle von den Gesundheitsfachberufen die Aufgaben übernommen werden können und an welcher Stelle die Diagnostik und Therapie über den Arzt angeordnet und geführt werden muss.

Gibt es zu dieser Wunschvorstellung auf ärztlicher Seite Widerstand? Immerhin geht es da ja um Kompetenzerweiterung.

Irene Maier: Würde ich bejahen. In der täglichen Praxis meist nicht; da wünscht man sich ein anderes Zusammenspiel. Aber auf der Funktionärsebene sind da noch etliche dicke Bretter zu bohren.

Das Interview führte Anne Marie Feldkamp, Bochum 